after the biomolecules have been isolated. "Once you have the samples and have extracted the biomolecules you are interested in, then you can perform quality control to determine if all your samples are similar," she says.

Even if the optimal time to fixation were found and standardized, this is only part of the problem; researchers still need to identify the best fixative to preserve both histology and biomolecules. Formalin, the most widely used fixative, provides good preservation of tissue histology but can cause problems for researchers interested in downstream molecular applications. "Formalin tends to crosslink the tissue to such an extent that it is hard to get fragments of DNA that are longer than, maybe, a hundred or two hundred base pairs," says Rimm. Formalin fixation causes the crosslinking of DNA as well as RNA and proteins, although proteins seem to fair better following formalin treatment (see 'Frozen in time').

\section{Preservation society}

Although tissue preservation has its problems, the good news is that these are now being dealt with. Governmental agencies, such as the US Office of Biorepositories and Biospecimen Research at the National Cancer Institute (NCI) in Bethesda, Maryland, are starting to tackle the difficult issue of biospecimen standardization. In June, the office released a guide to the NCI's best practices for biospecimen resources, detailing technical guidelines for NCI-supported biospecimen collection and storage. And researchers and companies are
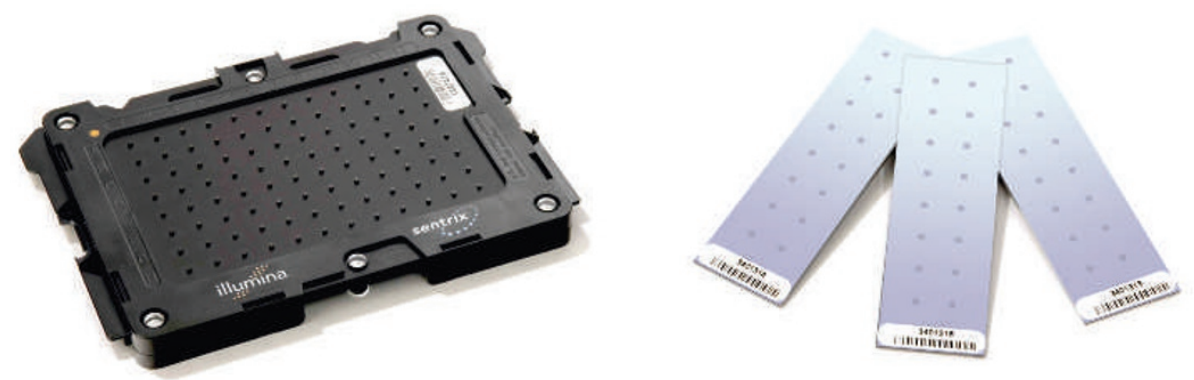

The DASL assay can be processed on two different platforms: the Array Matrix (left) or the BeadChip.

now creating methods to use the degraded and modified biomolecules obtained from FFPE samples for molecular analysis.

Isolation of RNA or DNA from FFPE tissue samples can be accomplished using a number of methods or kits. The main problem is that, almost without fail, the RNA or DNA isolated is degraded and chemically modified. But because researchers want to tap into the vast archives of FFPE tissues for global expression analysis and biomarker discovery, this is spurring companies to address the issue.

Microarray analysis has proven a valuable tool for understanding global gene-expression patterns. But using the degraded messenger RNA obtained from FFPE tissues for such analysis is problematic. "The results on standard microarrays are currently unsatisfactory," says Shawn Baker, scientific product manager for gene expression at Illumina in San Diego, California. For this reason, Illumina offers a geneexpression application that can study RNA extracted from FFPE tissue samples. Called DASL, short for cDNA-mediated annealing, selection, extension and ligation assay, this system amplifies the mRNAs from FFPEextracted samples, but unlike other amplification systems it is not 3' biased, says Baker. "We use a combination of random and oligo dT primers to generate the complementary DNA, which means that even with degraded RNA it still amplifies quite well and produces good, consistent profiles." Following the first amplification, the DASL system uses two gene-specific probes to amplify the cDNA. The resulting cDNA can be hybridized to a DASL-specific array. "The DASL assay is multiplexed up to 1,536 genes. So you get a tremendous boost in the overall throughput," says Baker.

Although unable to survey as many genes as standard microarrays, Baker says that using the DASL system, researchers have been able to profile FFPE tissue samples that are up to 30 years old - demonstrating the potential to

\title{
THE CUTTING EDGE
}

Researchers have the opportunity to play surgeon - slicing and dissecting out specific sections of tissue or even cell populations - with laser-capture microdissection (LCM). This level of analysis might seem to be difficult when applied to formalinfixed and paraffin-embedded (FFPE) tissue samples, but many companies are now offering easy and quick LCM solutions.

Leica Microsystems of

Wetzlar, Germany, offers the LMD6000 LCM system, which uses an upright microscope for dissection and capture. Christoph Horlemann, the company's product manager for the LMD6000, says this is possible because the transport mechanism for capture is based on gravity, unlike other LCM systems on the market.

'Transport mechanism' refers to the method for delivering a dissected tissue sample from a slide to a collection vessel. Other approaches to this process include that of PALM Microlaser Technologies in Bernried, Germany, which uses a 'pressure catapult' to send sections into tubes from the LCM instrument, and the CapSure system from Molecular Devices in Sunnyvale, California, which uses the laser to extend a polymer onto the tissue sample for capture.

Leica Microsystems' transport mechanism uses slides with a foil covering. The tissue is attached to the foil covering but not the slide, so researchers cut the tissue and the foil together and the sample simply falls by gravity into the collection tube below, explains Horlemann. But even this foil coating might not be needed in the future.

Both Leica and PALM are working with Expression Pathology of Gaithersburg, Maryland, on what may be

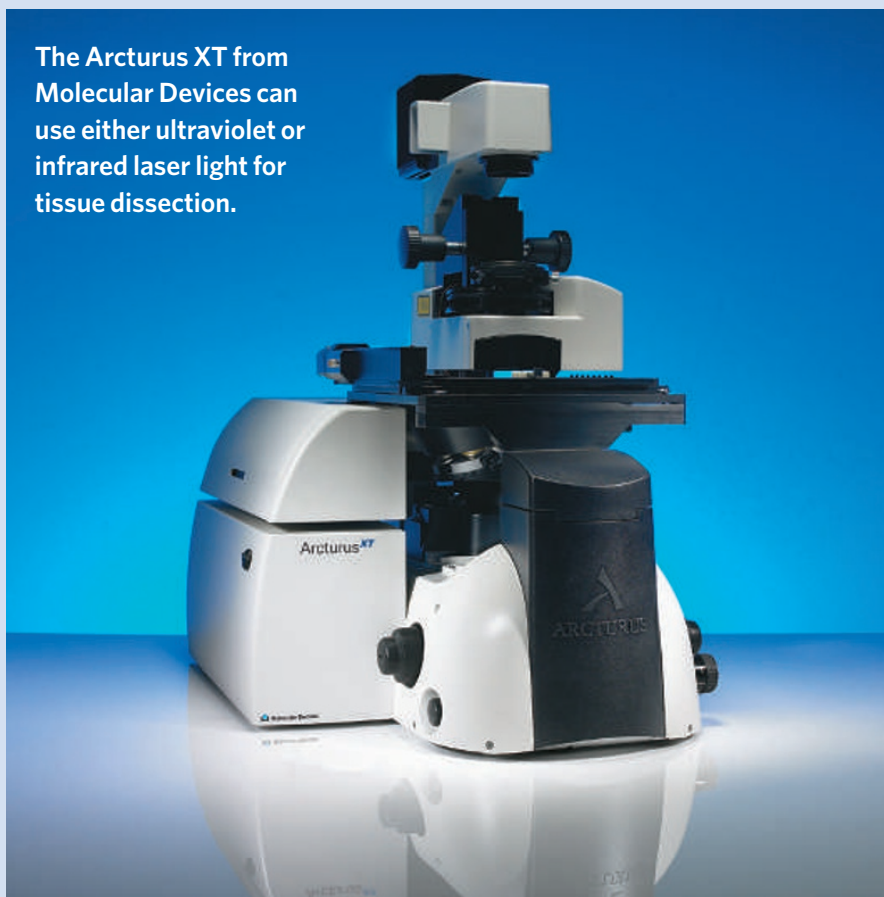

the next generation of LCM slides. Called Director, these glass slides are based on laserinduced forward transfer (LIFT), 
examine the numerous collections of archived FFPE samples. Illumina is now working to increase the number of genes analysed for each DASL assay.

NuGEN Technologies in San Carlos, California, is another company developing methods to use RNA extracted from FFPE tissue samples for gene-expression analysis. NuGEN specializes in working with very small amounts of RNA or difficult-to-use RNA, such as RNA extracted from whole blood or very degraded sources. This is commonly seen with clinical samples, but is most significant in the case of FFPE, says Gianfranco de Feo, senior director of customer solutions at NuGEN. Although NuGEN did not start off looking at RNA from FFPE tissue samples, it was the next logical step. "We have had a product on the market for over a year now that allows users to work with very degraded RNA in very limited amounts, down to 500 picograms. We built on that technology to create kits for the much more degraded RNA that comes from FFPE samples," says de Feo.

At the core of NuGEN's technology is its amplification and labelling system, which has been optimized to work with Affymetrix 3' microarrays. The system relies on a combination of random hexamers, similar to that of Illumina, augmented with oligo (dT) primers to convert mRNA into cDNA in a linear amplification process. The inclusion of oligo (dT) primers was essential because the Affymetrix arrays probe the ${ }^{\prime}$ ' ends of transcripts. But NuGEN hopes to have labelling and hybridization protocols and products for other microarray platforms available before the end of the year.

To determine how well these degraded RNA samples from FFPE tissue will work on microarrays, NuGEN developed a tool using quantitative real-time PCR (qPCR) assay. It turns out that the results from this assay correlate very well with the overall results of microarray analysis, says de Feo, allowing researchers to decide whether the data that could be obtained from the array will be of sufficient quality to continue. And this is critical information, as in some cases less than $50 \%$ of the transcripts on the array may hybridize with the amplified RNA.

\section{Think small}

Asuragen in Austin, Texas, is a new company on the commercial block, working to understand and characterize the biological role of small RNAs. Although founded only a year ago, Asuragen's RNA roots go much deeper. Asuragen is a spin-off of Ambion, a company that worked in the field of molecular biology with a focus on RNA for nearly 17 years. "At Ambion, we developed the first kits and technologies for characterizing small RNAs," says Gary Latham, associate director of technology development at Asuragen. "And when microRNAs emerged as a new class of regulatory RNAs in humans, we were sitting in an excellent position to explore this area of 'biological dark matter." In March 2006, Ambion was sold to Applied Biosystems for US\$273 million and a portion of those proceeds were used to fund Asuragen.

Asuragen has concentrated its efforts on the

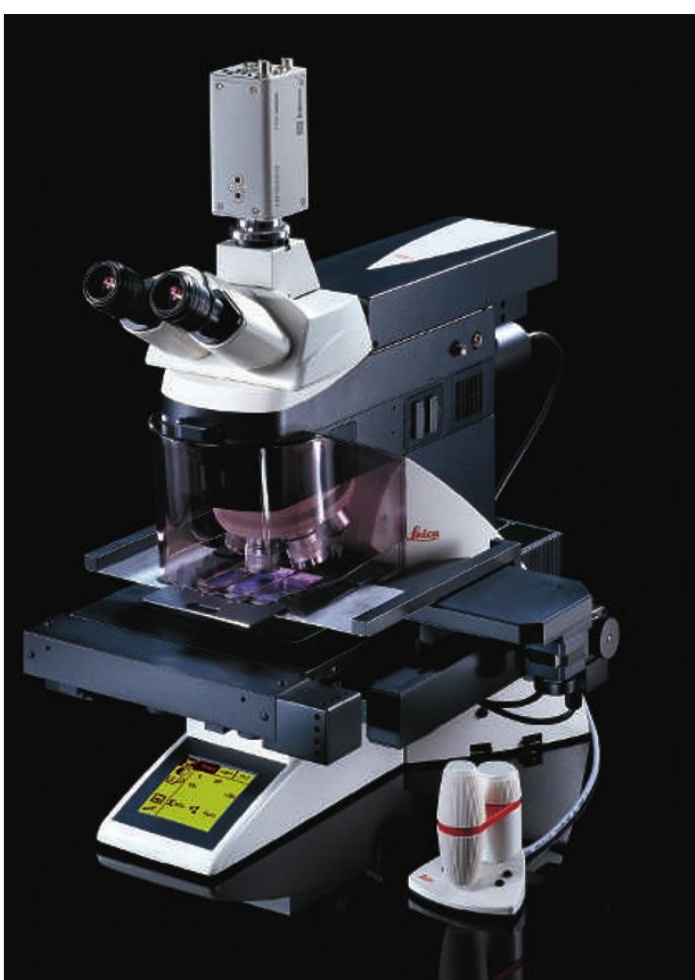

Leica Microsystems offers the LMD6000 for laser capture microdissection applications.

diagnostic and therapeutic opportunities of microRNAs (miRNAs). "As they are smaller, miRNAs tend to survive the more tortuous conditions of FFPE tissue processing better than mRNAs," says Latham, which makes a non-contact microdissection technique that uses a thin energytransfer coating that replaces plastic films or adhesives. The technology was co-developed by researchers at the US Naval Research Laboratory in Washington DC and scientists from Expression Pathology.

Laser energy is transferred to the transfer layer and the layer is vaporized. The laser energy is then converted into kinetic energy, and the selected feature is shot instantly into a collection tube.

LIFT works equally well whether the tissue is collected up (PALM) or down (Leica) as the energy is sufficient to propel the tissue into the collection tube either way. As the transfer layer completely absorbs the laser energy, the biomolecules in the sample are not affected. The use of glass with this coating has other advantages as well.

"Glass slides are quite useful as you can also perform fluorescence and contrast applications without any interfering foil," says Horlemann.

Molecular Devices, a division of MDS Analytical Technologies, acquired Arcturus in 2006 and is now providing both the Veritas and the Arcturus XT LCM systems. Whereas most LCM systems use an ultraviolet laser for cutting samples, the systems offered by Molecular Devices can have either ultraviolet or the standard infrared laser options.

"The infrared laser is ideal for small areas where a user is looking to pick up only a cell or two," says Steven Blakely, product manager for the Arcturus LCM systems. "Infrared allows for a gentle collection of cells."

The use of infrared laser light is critical to the CapSure system because the dye found in the CapSure polymer is activated and becomes adherent with infrared light. Blakely says the CapSure system is particularly useful for FFPE samples that are already mounted on glass slides because the transport mechanism involves only the polymer adhering to the tissue and so can work with any glass slide.

Cutting tissue samples with ultraviolet light offers advantages too, such as dissecting thicker samples. Leica has worked to optimize objectives for its LCM systems with high-energy ultraviolet-light transmission, allowing more power to come in direct contact with the tissue sample. Horlemann says this provides faster cutting of the thicker tissue samples while using less power.

The future of LCM might just lie in automation and increases in throughput. "Now, researchers want to do faster, automated microdissection for proteomics," says Horlemann. But before anything else, some hardware and software issues needed to be resolved.

Any automated software package has to control all microdissection steps including focusing the microscope, recognizing cells of interest, focusing the laser and defining the area to cut - definitely not a simple task, but one that companies have worked on and made tremendous strides in recent years. Horlemann says that new advances are making automation easier every day. He points to the Director slides, which can allow for contrasting methods and fluorescence, making it easier when attempting to automatically define cells of interest as a step in the right direction.

Although simpler now, further advances will be required before LCM becomes as easy as pushing a button and walking away. N.B. 